\title{
Discovery of Spatio-Temporal Patterns in Multivariate Spatial Time Series
}

\author{
GENE P. K. WU and KEITH C. C. CHAN, Department of Computing, The Hong Kong \\ Polytechnic University
}

\begin{abstract}
With the advancement of the computing technology and its wide range of applications, collecting large sets of multivariate time series in multiple geographical locations introduces a problem of identifying interesting spatio-temporal patterns. We consider a new spatial structure of the data in the pattern discovery process due to the dependent nature of the data. This article presents an information-theoretic approach to detect the temporal patterns from the multivariate time series in multiple locations. Based on their occurrences of discovered temporal patterns, we propose a method to identify interesting spatio-temporal patterns by a statistical significance test. Furthermore, the identified spatio-temporal patterns can be used for clustering and classification. For evaluating the performance, a simulated dataset is tested to validate the quality of the identified patterns and compare with other approaches. The result indicates the approach can effectively identify useful patterns to characterize the dataset for further analysis in achieving good clustering quality. Furthermore, experiments on real-world datasets and case studies have been conducted to illustrate the applicability and the practicability of the proposed approach.
\end{abstract}

CCS Concepts: $\bullet$ Mathematics of computing $\rightarrow$ Time series analysis; Cluster analysis; $\bullet$ Information systems $\rightarrow$ Data mining;

Additional Key Words and Phrases: Multivariate spatial time-series pattern discovery, spatio-temporal data mining, time-series clustering

\section{ACM Reference format:}

Gene P. K. Wu and Keith C. C. Chan. 2020. Discovery of Spatio-Temporal Patterns in Multivariate Spatial Time Series. ACM/IMS Trans. Data Sci. 1, 2, Article 11 (May 2020), 22 pages.

https://doi.org/10.1145/3374748

\section{INTRODUCTION}

Multivariate time series are generated massively due to recent developments in sensor and satellite technologies, medical measurements, climate informatics, and bioinformatics. These large-scale data encode important information about complex relations among individual time series. Recent works on multivariate time-series (MTS) pattern discovery focus mainly on extracting temporal association patterns and features [1-3]. Many of these MTS are spatio-temporal by nature in which they are collected together with location information such as latitude and longitude. For example, climate data are from sensors located in different regions, each of which collects periodic readings of variables such as humidity, wind speed, temperature, and rainfall intensity. Conventional timeseries analysis methods that consider only the time domain are often adopted to analyze MTS but

Authors' addresses: G. P. K. Wu and K. C. C. Chan, Department of Computing, The Hong Kong Polytechnic University, Hung Hom, Kowloon, Hong Kong; emails: \{gene.wu, keith.chan\}@polyu.edu.hk.

Permission to make digital or hard copies of all or part of this work for personal or classroom use is granted without fee provided that copies are not made or distributed for profit or commercial advantage and that copies bear this notice and the full citation on the first page. Copyrights for components of this work owned by others than the author(s) must be honored. Abstracting with credit is permitted. To copy otherwise, or republish, to post on servers or to redistribute to lists, requires prior specific permission and/or a fee. Request permissions from permissions@acm.org.

(C) 2020 Copyright held by the owner/author(s). Publication rights licensed to ACM.

2577-3224/2020/05-ART11 \$15.00

https://doi.org/10.1145/3374748 


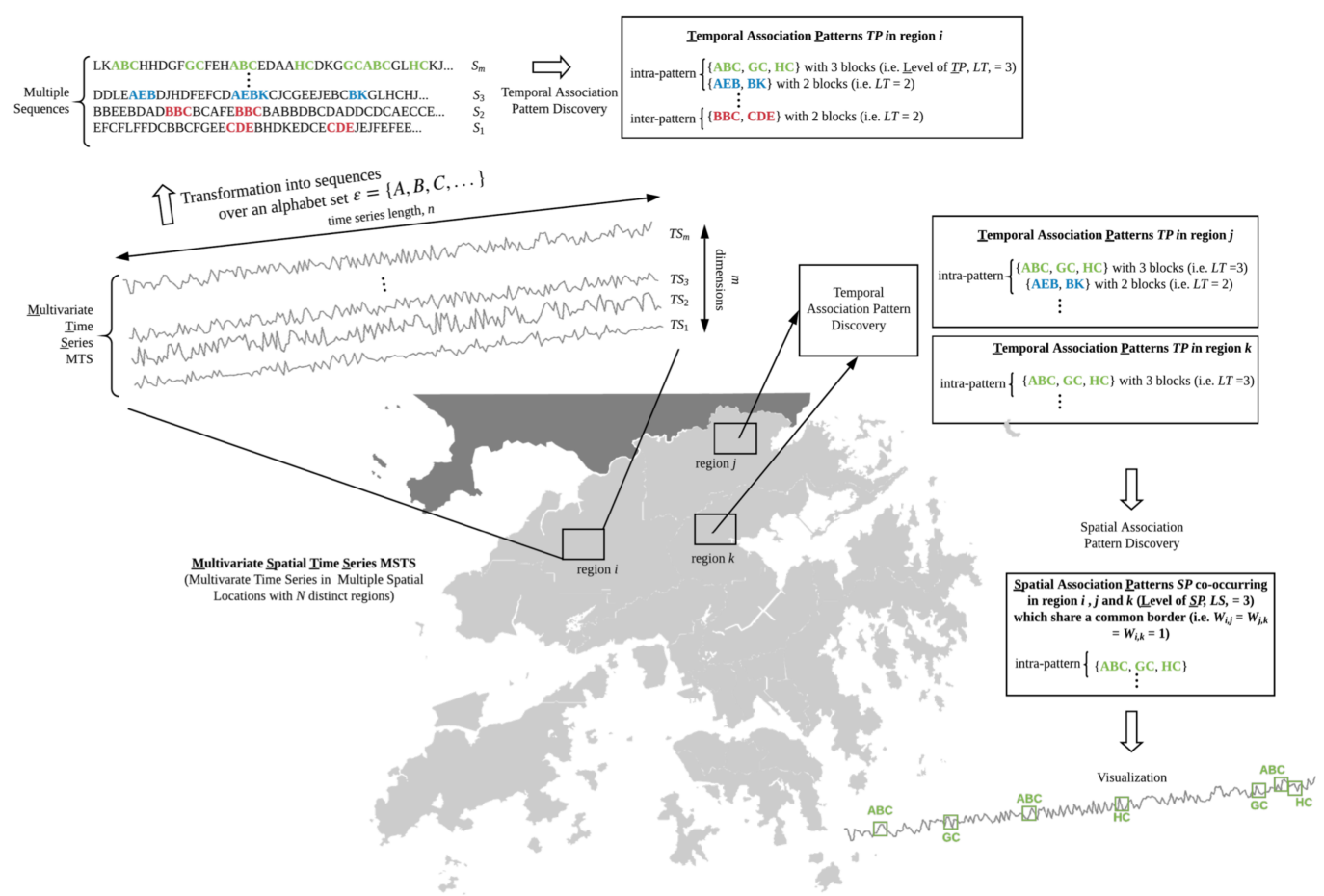

Fig. 1. Schematic of Multivariate Spatial Time-series pattern discovery.

relatively little works have been reported on discovering patterns in multivariate spatial time series (MSTS). A MSTS can be considered as made up of a collection of values by a set of geographical coordinates accompanied by a set of temporally interrelated variables monitored over a period of time at successive time instants spaced at uniform time intervals. In this regard, we focus on discovering patterns from MSTS.

This article proposes a general model for the problem of discovering patterns from MSTS. The model targets to tackle a specific spatio-temporal pattern mining task and is possible to tackle classification and clustering tasks after some modifications. We develop algorithms and evaluate the performance by comparing the proposed techniques with different traditional techniques on a simulated dataset and a real dataset. This proposed approach has been applied on three case studies to demonstrate the applicability and practicability. Our major contribution is threefold. (1) Classical spatial analysis studies entities using their topological, geometric or geographic properties. We define MSTS data structure, its associated temporal attributes, and its clustering problem. (2) Traditionally, analytical techniques favor the spatial definition of objects as points. We propose a general model to characterize MSTS. (3) Conventional approaches capture spatial dependency to provide information on spatial relationships in variable level. Our approach goes deeply to the attribute-value level.

As the motivation of the problem, let us begin with a simple application example of multivariate spatial time-series (MSTS) with three regions $(i, j, k)$ containing patterns $\{\mathrm{ABC}, \mathrm{GC}, \mathrm{HC}\},\{\mathrm{AEB}$, $\mathrm{BK}\}$, and $\{\mathrm{BBC}, \mathrm{CDE}\}$ as shown in Figure 1. The symbols in the patterns are from the alphabet set $\varepsilon$ due to the transformation of the time series (TS) into sequences (S). Here from Figure 1, this MSTS can be referred to as three multivariate time series (MTS) across three spatial locations. We can assume that the multivariate time series in each region are the climate variables such as precipi- 
tation, temperature, wind speed, and so on. The three selected regions are the regions of interest, which can be town centers in the study area. For application, finding the spatio-temporal association patterns in this study area can benefit the scientific officers to synthesize their observations into coherent explanations to draw inferences, e.g., rainfall analysis for typhoon in the weather forecast. In region $i$, every association pattern repeats 2 times and thus has occurrences of 2 . A pattern is made up of a collection of blocks in which each block contains a local part of the pattern occurring in a univariate time series. Pattern $\{\mathrm{ABC}, \mathrm{GC}, \mathrm{HC}\}$ has three blocks, pattern $\{\mathrm{AEB}, \mathrm{BK}\}$ has two blocks, and pattern $\{\mathrm{BBC}, \mathrm{CDE}\}$ has two blocks. The first and second patterns, known as intra-patterns, are along individual time series while the third pattern, known as inter-pattern, is across multiple time series. There are some varying time delays between the blocks in the patterns. These patterns occurring sequentially in time are referred to as temporal association patterns (TP). Thus, the number of blocks in a TP is also called the levels of TP (LT). Considering that temporal association pattern discovery is applied in other regions simultaneously, some temporal association patterns are also detected in region $j$ and region $k$. Among the $i, j$, and $k$ regions, pattern $\{\mathrm{ABC}, \mathrm{GC}$, $\mathrm{HC}$ s span across them significantly. This pattern is referred to as spatial association pattern (SP).

The discovered spatio-temporal association patterns, which may uncover previously unknown relations along and across individual time series in multiple spaces, could provide interesting insights for space- and time-dependent modeling and hypothesis generation in a variety of application domains such as climate, human activities, and public healthcare, in particular in environmental processes with a large number of sensors installed for process monitoring and analysis.

\section{RELATED WORK}

\subsection{Time Series}

Time-series value is numeric. In the real world, it is unusual to find time series that behave independently of others. Analyzing multiple time series simultaneously is an important task and is referred to as MTS analysis. MTS, which may carry a class label [4], can be considered as a collection of vectors. In the spatio-temporal context, MTS can exist in different locations and be referred to as MSTS. For example, climate data are MSTS recorded from several sensors to describe the weather by meteorological variables such as temperature, precipitation, humidity, wind speed at a given location and may come from multiple locations of either arid or semiarid climate types, labeled as "dry arid" and "dry semiarid."

\subsection{Time-series Analysis}

Although quite a number of studies have been conducted on time-series analysis methods, only a few of them focus on those with multivariate nature. A primitive approach proposed by Kruskal and Liberman [5] aims to find the longest common subsequence shared by MTS, but the algorithm grows exponentially in time with respect to the number of sequences concerned, making this approach undesirable against most of the possible applications. Later, Oates [6] came up with a method to discover "distinctive subsequence," in other words, "abnormal" pattern occurrences in a MTS. In this study, they randomly sampled the time series with length L sub-sequences and tried to discover patterns by clustering these subsequences based on Dynamic Time Warping. However, this study was restricted to discover distinctive subsequences but also did not consider the possible relations between different variables in the dataset.

\subsection{Multivariate Time-series Analysis}

A number of studies on MTS tried to utilize principle component analysis (PCA) to reduce the number of dimensions of the feature space in the dataset. PCA extracts principle components, 
which represent the most distinctive features of a MTS dataset. In 2001, Rosén and Yuan [7] utilized dynamic PCA to obtain principle components from different MTS, and then used fuzzy c-means to cluster the results obtained by PCA. This approach has been used by a lot of researchers for mining MTS and is effective when they treat a set of MTS as a single item and aim to cluster a number of these items. However, it lacks the ability to find patterns between variables in a MTS. Based on this, Yang and Shahabi [8] proposed a variant that uses PCA and singular value decomposition (SVD) to raise the precision of similarity measure between different MTS. Similar optimizations over PCAbased clustering have been proposed by others, such as Singhal and Seborg [9], using similarity factors based on PCA and Mahalanobis distance between datasets for similarity measure. Some work adopted Euclidean distance for similarity measure. PCA is also used in feature selection, as a preprocessing step in MTS analysis by Yoon et al. [10].

Hidden-Markov models (HMM) were used by Owsley et al. [11] to cluster MTS. This method focuses on clustering different MTS datasets and requires well-established $a$ priori information of initial classes. Zhou and Chan [12] proposed a model-based clustering algorithm to cluster MTS based on the discovered temporal patterns in each MTS and compare them with those discovered in the others so that MTS that exhibit similar patterns can be grouped together in the same cluster. This method discovers temporal patterns using confidence value (a.k.a lift ratio) to represent the relationship between different variables. It is application independent and can perform without any domain knowledge about relevant features or any assumption about underlying data models.

If the datasets of interest contain spatial information, then one could obtain more interesting results by mining such information. Coppi, D'Urso, and Giordani [13] altered the fuzzy c-means to incorporate spatial influence while clustering MTS. This method introduced a term called spatial penalty, influenced by the contiguity between different spatial units. Datasets that represent neighbors in space are more likely to be classified into the same cluster by this term. An application of clustering Italian provinces proved the effectiveness of it.

Shumway [14] used Kullback-Leibler discrimination and Chernoff information measure to calculate the disparity between two sets of MTS. They calculated the distance matrix based on the discrimination results of these two methods and used it for $k$-means clustering. A method of measuring linear and non-linear dependence between groups of MTS was proposed by [15]. This article also referenced other methods to measure relation between univariate time series.

While most of the studies reviewed above focus on clustering different MTS datasets, some studies emphasize on discovering patterns within a single dataset. Bünau et al. [16] came up with a method that breaks down a MTS dataset into stationary and non-stationary parts. Named "stationary subspace analysis," this method extracts stationary sources from non-stationary time series and was successfully applied to EEG data for extracting stationary patterns of brain activity. As for discovering common trends in MTS, Zuur et al. [17] proposed a method using EM algorithm to perform dynamic factor analysis that models data as trends, explanatory variables, and noise. This method could handle dataset with missing values or few data points, so it claimed to achieve superior applicability over its precedents. Another area in MTS analysis is the change point problem, which finds out the timestamps when the covariance structure of the series changes abruptly. Lavielle and Teyssière [18] proposed a method to solve this problem that outperforms previous methods.

There are studies conducted over other MTS topics. Tsay [19] tried to characterize and identify outliers. Frenzel and Pompe [20] applied partial mutual information to analyze coupling between time-series datasets. Amiri-Simkooei [21] analyzed noise in multivariate GPS time series.

In summary, previous studies on MTS focus on finding patterns either between different MTS datasets or within a single dataset. However, for a set of MSTS, it may be interested in both finding temporal patterns within a MTS and finding those across space. Moreover, conducting further 
correlation analysis over such spatio-temporal patterns might be able to unveil more useful knowledge.

\section{THE MULTIVARIATE SPATIAL TIME-SERIES PATTERN DISCOVERY PROBLEM}

In this section, we present an approach to mine MSTS. Given a set of MSTS, the proposed approach incorporates an effective initial MTS pattern mining algorithm [12] to detect temporal patterns in a set of MTS for each location. Then, we propose a new algorithm to detect co-occurrence of the discovered temporal patterns across locations by mining a transformed spatio-temporal pattern matrix (STPM) that characterizes the space to form spatio-temporal patterns. Furthermore, we effectively integrate this spatio-temporal pattern mining algorithm for classification and clustering. If the set of MSTS is labeled, then the discovered patterns can be weighted to support or against a certain class membership for the construction of a classifier. If the set of MSTS is unlabeled, then the discovered patterns in one location are compared against those discovered in the others so that MSTS that have similar discovered patterns are grouped together into the same cluster. In this article, we focus on spatio-temporal pattern discovery and the clustering to validate the effectiveness of the STPM representation, assuming MSTS is unlabeled.

\subsection{Definition and Notation}

Definition 3.1.1. Multiple spatial locations. Suppose there are multiple spatial locations $L=$ $\left\{l_{1}, \ldots, l_{|L|}\right\}$, each of which is represented by a region label and its set of geographic coordinates $g$ so that $L=\left\{\left(r_{1}, g_{1}\right), \ldots,\left(r_{|L|}, g_{|L|}\right)\right\}$, where each set of geographic coordinates $g$ contains longitude $x$ and latitude $y$ coordinates. $|L|$ is the number of locations in the study area. There are totally $N$ distinct regions $R=\left\{r_{1}, \ldots, r_{i}, \ldots, r_{N}\right\}$ that partition the locations of the study area and can be retrieved by a location-region mapping function $G(x, y)$ for a given longitude $x$ and latitude $y$ to return the respective region $r_{i}$. Some regions are connected, i.e., neighborhood regions. To represent the neighborhood relationship between regions, let $W$ be an adjacency matrix of region that assigns equal weights to all neighbors of regions, that is, $\{W\}_{i, j}=1$ if regions $r_{i}$ and $r_{j}$ share a common border or 0 otherwise. For each location, there exists at least 1 MSTS.

Definition 3.1.2. Multivariate spatial time series. A MSTS is a MTS with a longitude $x$ and latitude $y$. With $G(x, y)$, a MSTS can be converted to an MTS with a region label $r_{i}, i \in\{1 \ldots N\}$.

Definition 3.1.3. Multivariate time series. A MTS consists of $m$ individual time series TS = $\{1, \ldots, m\}$. A time series $T S$ is a finite sequence of real values $\left(v_{1}, v_{2}, \ldots, v_{n}\right)$ containing $n$ observations with unique time points $t p=\{1, \ldots, n\}$.

Definition 3.1.4. Sequence. A symbol sequence $S$ is a sequence of characters $s_{1}, s_{2}, \ldots, s_{n}$ over an alphabet set $\varepsilon$, where each $s_{i} \in \varepsilon$. $\varepsilon$ is a set of distinct characters with size $|\varepsilon| . n$ is the length of $S$. $S[i, j]$ is its substring from index $i$ to $j$. Each character represents an event, so $S$ can be called an event sequence. After discretization, a TS can be transformed into a symbol sequence $S$. SAX [22], a well-known discretization method for time-series data mining practitioners, is adopted here for discretization. $|\varepsilon|$ is a user-specified parameter that controls the granularity of the approximation. As its value increases, the diversity of characters increases, causing the total number of patterns to grow. Setting it too low, such as $<5$, will restrict the variety of the patterns. With too much diversity, the sample size for statistical significance will be underpinned. The choice of it has been analyzed extensively with the choice of $|\varepsilon|$ being $2,5,10,20$ in the context of temporal rule mining in [31]. It points out that no single parameter value can work well in all cases and is highly problem dependent. Although half of their experiments indicate the performance behaves similarly regardless of alphabet size, better accuracy can be achieved by more fine-grained alphabet. Therefore, 
we set it to 10 as the default choice. After discretization, an MTS can be transformed into a set of multiple symbol sequences $S_{1}, S_{2}, \ldots, S_{m}$.

Definition 3.1.5. Pattern. A pattern $P$ is a short sequence of consecutive characters $p_{1}, p_{2}, \ldots, p_{|P|}$ over $\varepsilon$, where $|P|$ is the length of the pattern. A pattern's length should be at least 2. Otherwise, each symbol in the alphabet set $\varepsilon$ is a pattern.

Definition 3.1.6. Pattern occurrence. A pattern $P$ is always associated with a symbol sequence $S$. $P$ occurs in an interval $[i, j]$ in $S$ if and only if $P=S[i, j] . o_{P}$ denotes the occurrence of $P$. All occurrences of $P$ are recorded in its occurrence list $L_{P}$ so $\left|L_{P}\right|$ is the number of occurrences of $P$ in $S$.

Definition 3.1.7. Frequent pattern. A frequent pattern is a pattern with its number of occurrences $\left|L_{P}\right|>\min _{o}$, where $\min _{o}$ specifies the minimum number of occurrences required. $\min _{o}$ is a user supplied parameter and is usually set to $0.03 n$ to $0.07 n$ by rules of thumb. The default setting of our selection is $0.05 n$, which is the midpoint of the range. A low-frequency threshold will introduce many redundant patterns of lengthy size since many repeating characters will be present in some sequences. Setting it too high, which will filter some weaker but important patterns, will produce too little samples that will undermine the statistical significance.

Definition 3.1.8. Temporal association of patterns. A temporal association pattern TP is an association of patterns occurring sequentially in time. Each pattern $P^{i}$ is a block of a TP. It describes the phenomenon that $P^{i+1}$ occurs within a certain specified time delay $t_{d}$ after $P^{i}$ occurs for $i=1, \ldots, L T-1$. [1] shows that this parameter is not sensitive in finding the associations. The default setting of $t_{d}$ is 6 to allow temporal patterns of various delays to be found. There are totally $L T$ blocks for a $T P$ and we call $L T$ level of $T P . \max _{L T}$ specifies the maximum level of $T P$. When all frequent patterns of a $T P$ are from the same sequence, $T P$ is called an auto association pattern or intra pattern. Otherwise, it is called cross association pattern or inter pattern. $|T P|$ is total number of temporal association patterns.

Definition 3.1.9. Temporal pattern occurrence. The set of occurrences of TP in the MTS is denoted by $L_{T P} .\left|L_{T P}\right|$ is the total number of occurrences.

Definition 3.1.10. Spatial association of patterns. A spatial association pattern $S P$ is an association of multiple temporal association patterns co-occurring in multiple regions. Each $T P^{i}$ is a building block of $S P$. It describes the phenomenon that $T P^{i+1}$ occurs in a region $r_{j}$ other than the region $r_{i}$ of $T P^{i}$, where $r_{i} \neq r_{j}$. There should be at least $2 T P$ s, i.e., 2 blocks, in a $S P$. Otherwise, every $T P$ is a $S P$. There are totally $L S$ blocks for $S P$, and we call $L S$ the level of $S P$. $\max _{L S}$ specifies the maximum level of $S P .|S P|$ is the total number of spatial association patterns.

Definition 3.1.11. Spatial association occurrence. The set of occurrences of SP in all MTS in different regions is denoted by $L_{S P} .\left|L_{S P}\right|$ is the total number of occurrences.

Definition 3.1.12. Statistical significance. The statistical significance based on adjusted residual [23] $d_{P}$ measures how significantly the observed frequency of occurrences of an association pattern $\left|L_{P}\right|$, which can be a $T P$ and/or a $S P$, deviates from its expected frequency $E_{P}$ adjusted by its variance $V_{P}$. It is given in Equation (3.1.12.1) as follows:

$$
d_{P}=\frac{\left(\left|L_{P}\right|-E_{P}\right) / \sqrt{E_{P}}}{\sqrt{V_{P}}}, P \in\{T P, S P\} .
$$


Definition 3.1.13. Weight of evidence. The weight of evidence measure provided by a pattern $p^{i}$ for or against the classification of an object $X$ into class $c_{i}$ is defined as:

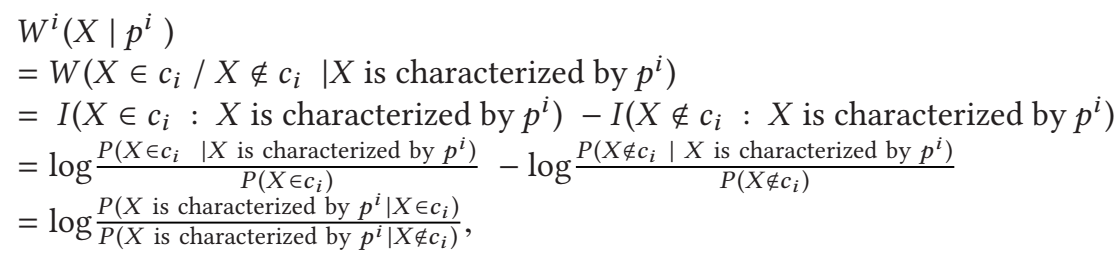

where $I()$ is the mutual information. It is positive if $p^{i}$ provides positive evidence supporting $X$ is classified to $c_{i}$. Otherwise, it is negative, or zero.

$P\left(X\right.$ is characterized by $\left.p^{i} \mid X \in c_{i}\right)$ is the probability that an object $X$ contains a pattern $p^{i}$ given that $X$ belongs to $c_{i}$. It is computed by counting the occurrence of objects in the database containing pattern $p^{i}$ and belonging to class $c_{i}$ divided by the number of objects belonging to class $c_{i}$.

$P\left(X\right.$ is characterized by $\left.p^{i} \mid X \notin c_{i}\right)$ is the probability that an object $X$ contains a pattern $p^{i}$ given that $X$ does not belong to $c_{i}$. It is computed by counting the occurrence of objects in the database containing pattern $p^{i}$ and not belonging to class $c_{i}$ divided by the number of objects not belonging to class $c_{i}$.

$W^{i}\left(X \mid p^{i}\right)$ can be interpreted as a measure of the difference in the gain in information when an object $X$ containing $p^{i}$ is classified into $c_{i}$ as opposed to other classes. $W^{i}\left(X \mid p^{i}\right)$ is positive if $p^{i}$ provides positive evidence supporting the classification of $X$ into $c_{i}$. Otherwise, it is negative.

Given the interesting patterns $P=\left\{p_{1}^{1}, \ldots, p_{j}^{i}, \ldots, p_{m_{i}}^{|C|}\right\}$, discovered for each corresponding $|C|$ classes, $c_{1}, \ldots, c_{i}, \ldots, c_{|C|}$, an unseen object $X_{u}$ can be classified by matching it against the patterns in each of classes. An unseen MSTS object $X_{u}$ is first transformed into a list of attributes using the proposed STPM to construct a set of patterns $P^{u}$ for matching. Then for every pattern $p_{j}^{i}$ that $X_{u}$ matches, there is some evidence $W^{i}\left(X_{u} \mid p_{j}^{i}\right)$ provided by it for or against the classification of $X_{u}$ into $c_{i}$. Assuming that $X_{u}$ matches with $n_{i} \leq m_{i}$ patterns in $P$ of $c_{i}$, we calculate a total weight of evidence measure for $X_{u}$ to be classified into $c_{i}$.

Definition 3.1.14. Total weight of evidence. The total weight of evidence provided by each of individual patterns is a measure for $X_{u}$ to be classified into $c_{i}$ and is defined as

$$
\begin{aligned}
& W^{i}\left(X_{u}\right) \\
& =W\left(X_{u} \in c_{i} / X_{u} \notin c_{i} \mid X_{u} \text { is characterized by } p_{1}^{i}, \ldots, p_{j}^{i}, \ldots, p_{m_{i}}^{i}\right) \\
& =\sum_{j=1}^{m_{i}} W\left(X_{u} \in c_{i} / X_{u} \notin c_{i} \mid X_{u} \text { is characterized by } p_{j}^{i}\right)
\end{aligned}
$$

\subsection{Problem Formulation}

Given the parameter $|\varepsilon|$ for discretization of sequence and bounding parameters $\min _{o}, t_{d}, \max _{L T}$, $\max _{L S}$, we aim at discovering interesting spatial and temporal associations from multiple time series in multiple locations. We provide a detail discussion about the setting of parameters in the definition above and some subsequent experiments below.

\subsection{Temporal Association Pattern Discovery}

The temporal association pattern discovery is outlined in this section and represented as pseudocode in Algorithm 1 as follows. 


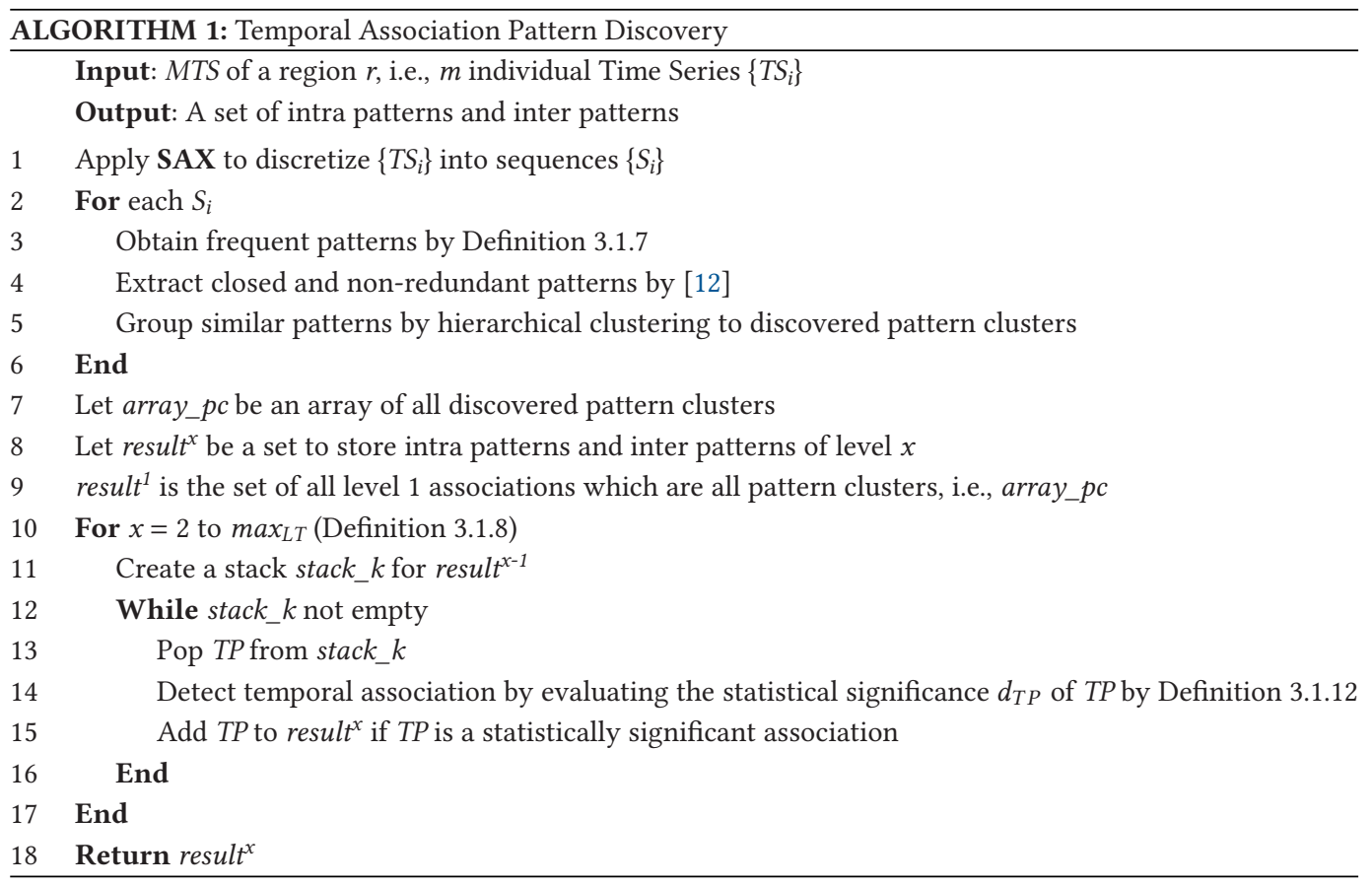

After SAX transformation of MTS into multiple symbol sequences, we discover frequent patterns (Definition 3.1.7) from each sequence. To reduce the number of frequent patterns, we prune them by adopting the algorithms proposed in Reference [12] to effectively extract closed and nonredundant patterns.

Once all non-redundant patterns are extracted, a hierarchical clustering algorithm, which repeatedly groups the most similar pair of clusters into a new cluster until forming a single cluster with all objects, with an appropriate priority queue to improve the runtime is used to group similar patterns to further reduce the number of patterns. After this, by updating the occurrence list $L_{T P}$, a new occurrence list $L_{C}$ is created for each cluster of patterns by taking the union of all occurrences of patterns in the cluster.

After all pattern clusters, treated as the building blocks for each sequence, are discovered, we can detect temporal associations (Definition 3.1.8) among them based on statistical significance (Definition 3.1.12). It forms pattern clusters for each sequence first to yield intra patterns and grows temporal associations level by level to yield inter patterns. To compute the statistical significance $d_{T P}$ for temporal association patterns and given the above definitions to count the observed frequency of occurrences of association patterns, we want to estimate the expected frequency of occurrences of association patterns and its variance. Let $T P^{\prime}$ be the temporal association composed of $L T-1$ blocks of $T P$ and $P^{L T}$ be the last block of $T P$. Assuming that the last block occurs randomly at a position in its respective sequence with probability estimated as $p=\frac{\left|L_{P L T}\right|}{|S|}$, for temporal association of patterns, we take the association of $T P^{\prime}$ with $P^{L T}$ as the estimate of the expected frequency of occurrences of TP. Then, the expected frequency of occurrences $E_{T P}$ for $T P$ is calculated as $\operatorname{Pr}\left(\gamma^{\prime} \vee \rho^{L T}\right) \cdot\left|L_{T P^{\prime}}\right|$ and variance $V_{T P}=E_{T P} \cdot\left(1-\operatorname{Pr}\left(\gamma^{\prime} \vee \rho^{L T}\right)\right)$, where $\gamma^{\prime}$ is an occurrence of, $\rho^{L T}$ is an occurrence of $P^{L T}$ and $\operatorname{Pr}\left(\gamma^{\prime} \vee \rho^{L T}\right)$ is the probability that $\gamma^{\prime}$ is associated with $\rho^{L T}$. $\operatorname{Pr}\left(\gamma^{\prime} \vee \rho^{L T}\right)$ is calculated as $1-(1-p)^{t_{d}+1}$. After all temporal association patterns in a region $r_{i}$ are discovered, the above procedure repeats until all regions $R$ are processed. 
Table 1. Spatio-Temporal Pattern Matrix (STPM)

\begin{tabular}{|l|l|l|l|l|l|}
\hline$d_{T P}$ & $A_{1}$ & $\ldots$ & $A_{j}$ & $\ldots$ & $A_{M}$ \\
\hline$X_{1}$ & $d_{11}$ & $\ldots$ & $d_{1 j}$ & $\ldots$ & $d_{1 M}$ \\
\hline$\ldots$ & $\ldots$ & $\ldots$ & $\ldots$ & $\ldots$ & $\ldots$ \\
\hline $\boldsymbol{X}_{\boldsymbol{i}}$ & $d_{i 1}$ & $\ldots$ & $d_{i j}$ & $\ldots$ & $d_{i M}$ \\
\hline$\ldots$ & $\ldots$ & $\ldots$ & $\ldots$ & $\ldots$ & $\ldots$ \\
\hline $\boldsymbol{X}_{\boldsymbol{N}}$ & $d_{N 1}$ & $\ldots$ & $d_{N j}$ & $\ldots$ & $d_{N M}$ \\
\hline
\end{tabular}

\subsection{Spatio-Temporal Pattern Matrix Representation (STPM)}

For all regions $R=\left\{r_{1}, \ldots, r_{\mathrm{N}}\right\}$, putting all temporal association patterns together, we can form the final group of $M$ temporal association patterns (TP's), where $M=R \times M^{\prime}$ and $M^{\prime}$ is the number of discovered TP's in each region $r_{i}$. Here, $d_{T P}$ refers to as statistical significance $d_{P}$ of $T P$. We can generate an $M$-dimensional feature space and transform each region $r_{i}$ into a vector $X_{i}$ in the $M$-dimensional feature space. Each vector $X_{i}$, where $i=1, \ldots, N$, is then characterized by $M$ attributes, denoted as $A=\left\{A_{1}, \ldots, A_{j}, \ldots, A_{M}\right\}$, whose values $d_{i 1}, \ldots, d_{i j}, \ldots, d_{i M}$, where $d_{i j}$ (Definition 3.1.12) represents the amount and the statistical significance of association patterns found in the $j$ th attribute in region $i$. We call this representation a spatio-temporal pattern matrix (STPM) that characterizes the region by the discovered TP's. To better visualize STPM, a table formatted with $N$ rows and $M$ columns (Table 1) is used. The discovered TP's of each region (record) are the columns (attributes) in the table. Sorting the column by $d_{T P}$ values can rank them based on their statistical significance.

\subsection{Spatial Association Pattern Discovery}

The spatial association pattern discovery is outlined in this section and represented as pseudocode in Algorithm 2 as follows.

After transforming the MSTS into STPM, we detect interesting association between temporal associations across regions. To do so, we first discretize the values of $d_{T P}$ based on the unsupervised algorithm described in Reference [24], which uses an information measure that reflects interdependence to group attributes and identify the representative attribute in each attribute group to drive the discretization. For now, each attribute $A_{j}$ contains only interval event values denoted as $A_{j}=\left\{A_{j}^{b} \mid b=1, \ldots, B\right\}$, where $B$ is the number of bins. Optimizing the discretization is not the issue addressed here, so we will not further discuss it.

To detect association, we construct a contingency table to count occurrences of values between 2 attributes, i.e., $A_{j}^{p}$ is $p t h$ value of $j t h$ attribute and $A_{j^{\prime}}^{k}$ is $k$ th value of the $j^{\prime}$ th attribute, $j \neq j^{\prime}$. Let $o_{p k}$ be the total number of occurrences when $A_{j}=A_{j}^{p}$ and $A_{j^{\prime}}=A_{j^{\prime}}^{k} ; e_{p k}=\frac{1}{T} \sum_{i=1}^{B} o_{p i} \sum_{i=1}^{B^{\prime}} o_{i k}$, where $\sum_{i=1}^{B} o_{p i}$ is the total number of counts when $A_{j}=A_{j}^{p}, \sum_{i=1}^{B^{\prime}} o_{i k}$ is the total number of counts when $A_{j^{\prime}}=A_{j^{\prime}}^{k}$, and $T$ is the number of records. With $o_{p k}$ and $e_{p k}$, we can detect whether or not $o_{p k}$ is significantly different from $e_{p k}$ by adjusted residual (Definition 3.1.12) substituting $\left|L_{P}\right|$ and $E_{P}$ by $o_{p k}$ and $e_{p k}$ respsectively to calculate $d_{p k}$. To reveal statistical significance, at $95 \%$ confidence level, if $d_{p k}>1.96$, then we can conclude it is a positive association; if $d_{p k}<-1.96$, then it is a negative association; if $-1.96<d_{p k}<1.96$, it is random. Table 2 visualizes the contingency table. According to Definition 3.1.10, for $d_{p k}>1.96, A_{j}^{p}$ and $A_{j^{\prime}}^{k}$ should come from different regions for their association to be considered as a SP. This SP and its occurrences $o_{p k}$ will be added to $L_{S P}$ (Definition 3.1.11). 
Table 2. Contingency Table of $A_{j}$ and $A_{j^{\prime}}$

\begin{tabular}{|c|c|c|c|c|c|c|}
\hline$A_{j^{\prime}}$ & $A_{j}^{1}$ & $\cdots$ & $A_{j}^{p}$ & $\cdots$ & $A_{j}^{B}$ & Total \\
\hline$A_{j^{\prime}}^{1}$ & $\begin{array}{c}o_{11} \\
\left(e_{11}\right)\end{array}$ & $\ldots$ & $\begin{array}{c}o_{p 1} \\
\left(e_{p 1}\right)\end{array}$ & $\cdots$ & $\begin{array}{c}o_{B 1} \\
\left(e_{B 1}\right)\end{array}$ & $o_{+1}$ \\
\hline$\cdots$ & . & "'. & '." & ". & '." & "'. \\
\hline$A_{j^{\prime}}^{k}$ & $\begin{array}{c}o_{1 k} \\
\left(e_{1 k}\right)\end{array}$ & $\cdots$ & $\begin{array}{c}o_{p k} \\
\left(e_{p k}\right)\end{array}$ & $\cdots$ & $\begin{array}{c}o_{B k} \\
\left(e_{B k}\right)\end{array}$ & $o_{+k}$ \\
\hline$\cdots$ & & $\cdots$ & $\cdots$ & $\cdots$ & $\cdots$ & $\ldots$ \\
\hline$A_{j^{\prime}}^{B^{\prime}}$ & $\begin{array}{c}o_{1 B^{\prime}} \\
\left(e_{1 B^{\prime}}\right)\end{array}$ & $\cdots$ & $\begin{array}{c}o_{p B^{\prime}} \\
\left(e_{p B^{\prime}}\right)\end{array}$ & $\cdots$ & $\begin{array}{c}o_{B B^{\prime}} \\
\left(e_{B B^{\prime}}\right)\end{array}$ & $o_{+B^{\prime}}$ \\
\hline Total & $o_{1+}$ & $\ldots$ & $o_{p+}$ & $\ldots$ & $o_{B+}$ & $T$ \\
\hline
\end{tabular}

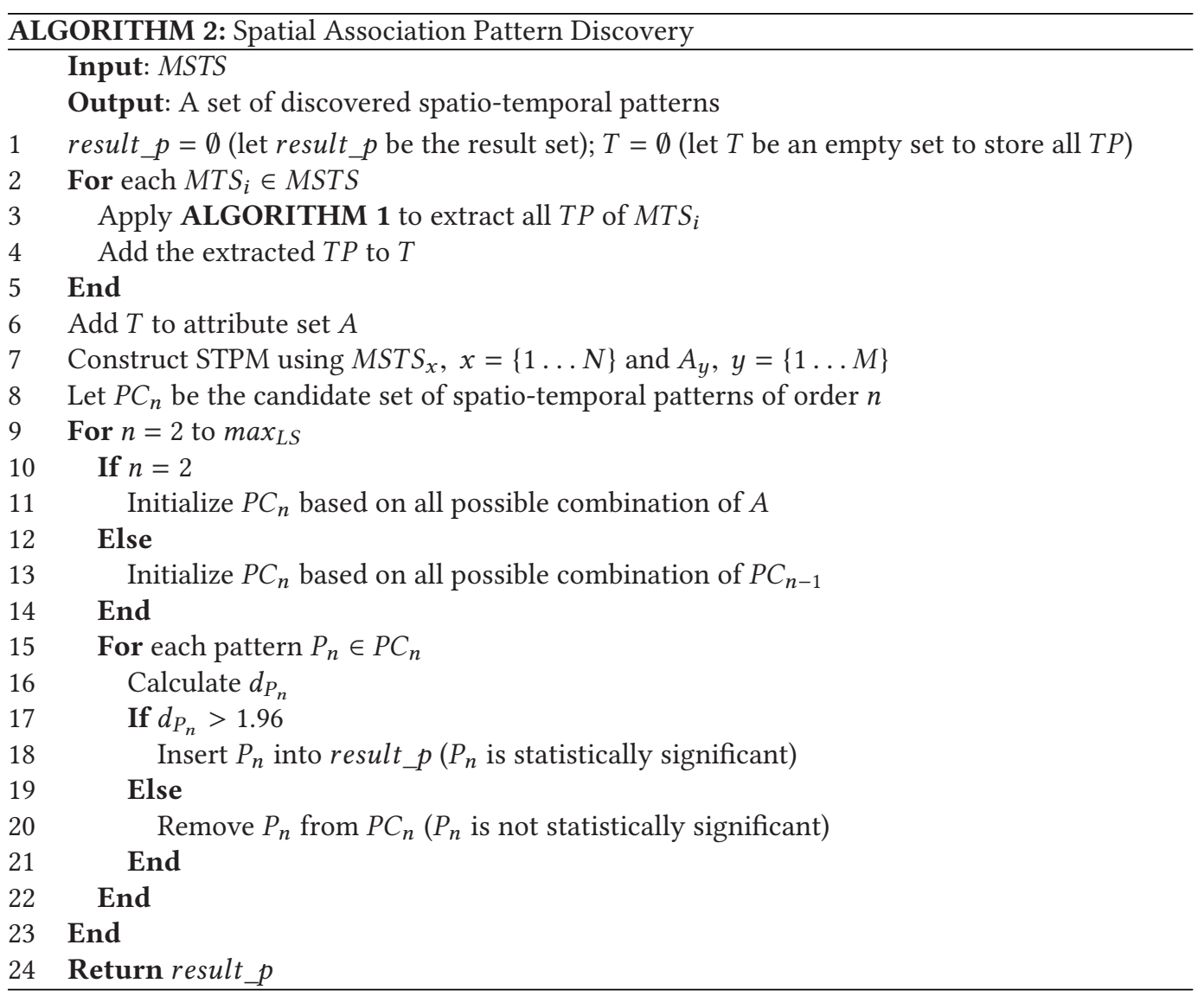




\subsection{Clustering and Re-Clustering Spatio-Temporal Pattern Matrix}

With STPM, it is readily available to perform cluster analysis on the set of $d_{T P}$ by treating each vector $X_{i}$, characterized by $M$ attributes, as an object by the state of the art clustering algorithm. The proposed clustering approach consists of 2 stages, initial clustering and re-clustering. The initial clustering stage, which locally optimizes the clustering, assigns cluster labels to the objects. We believe a good clustering result should generate good cluster label, so we treat the cluster label as the class label to perform classification so as to globally partition the objects. This re-clustering stage basically is to fit a clustered STPM into a classifier.

For initial clustering, we adopt a popular agglomerative hierarchical clustering to repeatedly group the most similar pair of clusters into a new cluster until forming a single cluster with all objects. It is optional to apply a suitable cutoff level to obtain a specific number of clusters based on prior knowledge. Pearson correlation coefficient is used for distance measure rather than Euclidean distance so as to be better in dealing with noise [25]. For a pair of objects $X_{i}$ and $X_{j}$ with values of $M$ attributes, the similarity measure is defined as $\operatorname{Sim}\left(X_{i}, X_{j}\right)=\frac{M \sum_{k=1}^{M} d_{i k} d_{j k}-\sum_{k=1}^{M} d_{i k} \sum_{k=1}^{M} d_{j k}}{\sqrt{M \sum_{k=1}^{M} d_{i k}{ }^{2}-\left(\overline{d_{i}}\right)^{2}} \sqrt{M \sum_{k=1}^{M} d_{j k}^{2}-\left(\overline{d_{j}}\right)^{2}}}$. To reflect the contiguity between different spatial units, we introduce $W_{i, j}$ (Definition 3.1.1) and add it as a term to $\operatorname{Sim}\left(X_{i}, X_{j}\right)$ as the spatial penalty. This forms a new similarity measure $\operatorname{Sim}^{\prime}\left(X_{i}, X_{j}\right)$, i.e., $\operatorname{Sim}^{\prime}\left(X_{i}, X_{j}\right)=\operatorname{Sim}\left(X_{i}, X_{j}\right)+W_{i, j}$, for clustering.

Treating the assigned cluster label in initial clustering as the class label, let $p^{i}$ be an interesting pattern discovered from class $i, c_{i}$. In a supervised manner, if the interesting pattern $p^{i}$ is conditioned by the class label $c_{i}$, it can be treated as a classification rule [26], i.e., if \{antecedent or left-hand-side or LHS\}, then \{consequent or right-hand-side or RHS\}. The weight of evidence measure $W$ (Definition 3.1.13) in information theory [26] is used to quantify the evidence of the joined significant rules to support or against a certain class membership. An example rule for classifying a MTS is if $\{$ temporal pattern $1=$ high and temporal pattern $2=$ low $\}$, then $\{$ class $=1\}$ with a weight of evidence of a certain value.

The task of classification is to maximize the total weight of evidence $W^{i}\left(X_{u}\right)$ (Definition 3.1.14). The total weight of evidence for $X_{u}$ to be classified into each of $c_{1}, c_{2}, \ldots, c_{|C|}$ is computed and $X_{u}$ is assigned to the class that can give the highest total weight of evidence. This measure is able to differentiate the case that when some identical objects refer to different classes in the training set as the class, assignment of $X_{u}$ is by the highest total weight of evidence.

\subsection{Complexity Analysis}

For the temporal association pattern discovery method of each region, the runtime and space complexity is $O\left(m^{L T} n^{L T+1} t_{d}^{L T-1}\right)$, where $m$ is the number of time series, $n$ is the number of time points, $L T$ is the level of the associations, and $t_{d}$ is the time delay. Now, for the spatio-temporal association pattern discovery, we have the STPM for all regions $R=\left\{r_{1}, \ldots, r_{N}\right\}$ forming a $R \times M^{\prime}$ matrix and let $k$ be the number of distinct cluster labels. To compute the contingency table for all $\left(\begin{array}{c}M^{\prime} \\ 2\end{array}\right)$ pairs of discretized attributes, the computational complexity is $O\left(M^{\prime 2} N\right)$. For $i>2$, to generate $i$ th- order spatio-temporal patterns, the computational complexity is $O\left(M^{\prime}{ }^{\prime} N\right)$. For higher-order patterns, the candidate set is greatly reduced by previous iterations, as the algorithm only considers growing statistically significant patterns, so all pattern candidate set should be much less than all possible combinations of $i$ th-order patterns, i.e., $\left|C_{i}\right|<M^{\prime}{ }^{i}$. If we predefine the number of discretized attributes, then the overall computational complexity is linear in terms of $N$. The initial clustering scans through the entire feature matrix once, so the computational complexity of calculating the pairwise similarity is $O\left(M^{\prime} N^{2}\right)$. For the re-clustering, it calculates the weight of evidence of all individual interesting patterns supporting or refusing the classification of an object 
into a class. Given $Q$ interesting patterns and $k$ distinct cluster labels, it calculates $k Q$ times. Each time scans through the entire database of $N$ regions. Consequently, it takes $Q N k$ operations to obtain all weights of evidence between each interesting pattern and each class. Hence, its computational complexity is $O(k N Q)$. The classification of an unseen MSTS requires the generation of $M^{\prime}$ discretized attributes from its MTS and match them against $Q$ interesting patterns to look for the highest total weight of evidence to support the classification into a certain class. As a result, it takes $k M^{\prime} Q$ operations in total for which the computational complexity is $O\left(k M^{\prime} Q\right)$ for the prediction of class.

\section{EXPERIMENTS AND EVALUATION}

In summary, we have performed both experimental and case studies of the proposed algorithms. Extensive experiments on synthetic data, real data, and case studies have been conducted. In all of the below experiments and case studies, we set $|\varepsilon|=10, t_{d}$ to $6, \max _{L T}=2$, and $\max _{L S}=5$ unless we re-specify these values. These values are chosen based on the domain knowledge and experiment setting. Other parameters will be specified in each experiment section.

\subsection{Experiments on Synthetic Dataset}

In this experiment, we generated MSTS for each of 60 regions, i.e., 60 MSTS, which belong to 3 clusters $\left\{C_{1}, \ldots, C_{3}\right\}$ each of which contains 20 MTS. For MSTS in the same cluster, they share common borders, i.e., $\{W\}_{i, j}=1$ for region $r_{i}$ and $r_{j}$ sharing a common border and in the same cluster or 0 for region $r_{i}$ and $r_{j}$ in different cluster. For each location, there exists at least 1 MSTS. Each MTS consists of $4 T S$, i.e., $\left\{T S_{1}, \ldots, T S_{4}\right\}$. To simulate the spatio-temporal relation, we implant patterns to regions sharing the common borders, i.e., same cluster. For regions that are spatially connected by the common border, they should contain more similar temporal patterns than those regions that are not connected. For each TS in each cluster, intra-patterns and inter-patterns are embedded so that MTS within the same cluster contains more similar patterns than those from others. Each TS contains 200 time points and can take on real values from 0 to 1. For $C_{1}, T S_{1}$ 's values are uniformly distributed within $[0,1]$; $T S_{2}$ 's values are uniformly distributed within $[0.18,0.44]$ in every 5 time point interval stochastically or otherwise uniformly distributed within $[0,1] ; T S_{3}$ takes on values uniformly distributed within $[0.03,0.39] ; T S_{4}$ takes on values uniformly distributed within $[0.32,0.49]$ if $T S_{3}$ 's value falls into $[0.18,0.39]$. For $C_{2}$, $T S_{2}$ 's values are uniformly distributed within $[0,1]$; if $T S_{2}$ 's value falls into $[0.18,0.44]$, the corresponding value in $T S_{1}$ is uniformly distributed within [0.53, 0.86]; if $T S_{2}$ 's value falls into [0.40, $0.66]$, the corresponding value in $T S_{4}$ is uniformly distributed within $[0.23,0.41]$ at the next time point in $60 \%$ chance and the corresponding value in $T S_{3}$ is uniformly distributed within [0.05, 0.49] at the next time point in $40 \%$ chance. For $C_{3}$, values of $T S_{1}$ and $T S_{3}$ are uniformly distributed within $[0,1]$; if $T S_{3}$ 's value falls into [0.03, 0.22], the corresponding value in $T S_{2}$ is uniformly distributed within $[0.53,0.92]$; if $T S_{1}$ 's value falls into $[0.70,0.90]$ at every six time points, $\mathrm{TS}_{4}$ takes on a value uniformly distributed within $[0.13,0.47]$ at the next time point. Figure 2 shows the MSTS of the three clusters with the implanted patterns highlighted. In this experiment, we set $\min _{o}$ to 10 , which is $5 \%$ of 200 time points, based on the observation of the generated data.

This set of experiments aims at demonstrating the ability in recovering the embedded spatiotemporal association patterns. A good pattern discovery algorithm should produce, not too many, high-quality patterns that can find the implanted associations. Therefore, to compare the number of generated association patterns, we use the approach developed by Tatavarty et al. [27], namely TARM, to generate temporal association patterns. With these generated temporal patterns, the STPM is constructed so we can discover spatio-temporal patterns for comparison. Second, we 


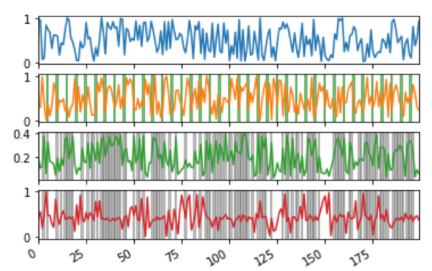

MSTS in Cluster 1

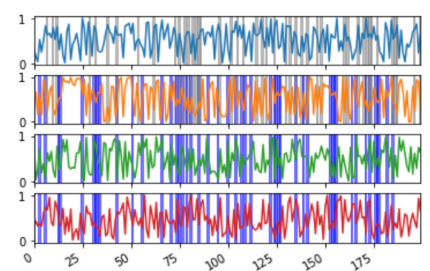

MSTS in Cluster 2

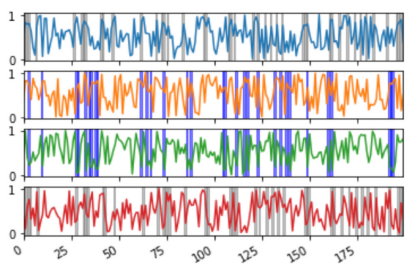

MSTS in Cluster 3

Fig. 2. Synthetic MSTS with implanted patterns highlighted.

also believe that a good representation of the transformed data can lead the clustering algorithms to produce promising clustering results so we applied three different model-based approaches to transform the MSTS to STPM for cluster analysis.

After running TARM and then the construction of the STPM, we detect the generated spatiotemporal association patterns and compare it with the one generated by the proposed algorithm. The proposed algorithm generated 73,587 temporal associations and completely captures the implanted temporal associations. The discovered temporal associations by the proposed approach and TARM are ranked by statistical significance and confidence respectively. TARM generated $1,177,424$ temporal associations and only up to the $74,976^{\text {th }}$ ranked association captures 12 occurrences of the implanted association. To explore the efficiency of the proposed algorithm by varying the parameter sizes of $\max _{L T}$ and $\max _{L S}$, we created two study groups: Study Group A, $2 \leq \max _{L T} \leq 3$ and $\max _{L S}=3$, and Study Group B, $2 \leq \max _{L T} \leq 5$ and $\max _{L S}=3$. A desktop computer with $32-$ GB RAM is used to run these experiments. In study group A, while TARM, which took around 65 minutes, generated 2,648,487 temporal associations and can never finish running spatial discovery due to running out of memory and generating too many temporal associations, the proposed algorithm, which took $97 \mathrm{~s}$, generated 113,861 temporal associations and 923 spatio-temporal associations. In study group B, TARM ran out of memory in generating both temporal and spatial associations while it took around 158 seconds for the proposed algorithm to execute the program to generate 152,118 temporal associations and 1,189 spatio-temporal associations. Therefore, the STPM-based on the patterns generated from TARM is very sparse and is not feasible for further cluster analysis. It is very time consuming to expand the associations to the maximum level of $L T$ and $L S$ for TARM and the visualization of the result is not human readable for data exploration. We consider limiting $\max _{L T}<L T-1$ and $\max _{L S}=L S$. The proposed method outperforms TARM by generating smaller number of temporal associations with better ranking of the discovered temporal patterns that cover the embedded ones. To assess the scalability of the proposed algorithm, we increased the $\max _{L T}$ a number of times to grow the size of the problem (i.e., searching more higher-order patterns) in the experiment to evaluate if the runtime of the proposed algorithms increases in a scalable manner. Figure 3 plots the results of the scalability test. It indicates that the increase of maximum levels of $L T$ results in the increase of runtime. For the increase of $\max _{L T}$, there is a small linear growth in the number of temporal association patterns, but we benefit from the logarithmic scale of runtime for larger $\max _{L T}$. We go on searching the spatio-temporal associations from the STPM. For the proposed approach, 710 spatio-temporal association patterns are detected. Ranked by the statistical significance, the top 9 patterns are depicted in Table 3 for reference. For the top 399 of 710 patterns, it is interesting to note that none of them are associated with different clusters so they are likely to describe the characteristics and the cluster relationship without relying too much on the spatial contiguity information. Thus, identifying such spatio-temporal associations in MSTS can aid in quicker and 


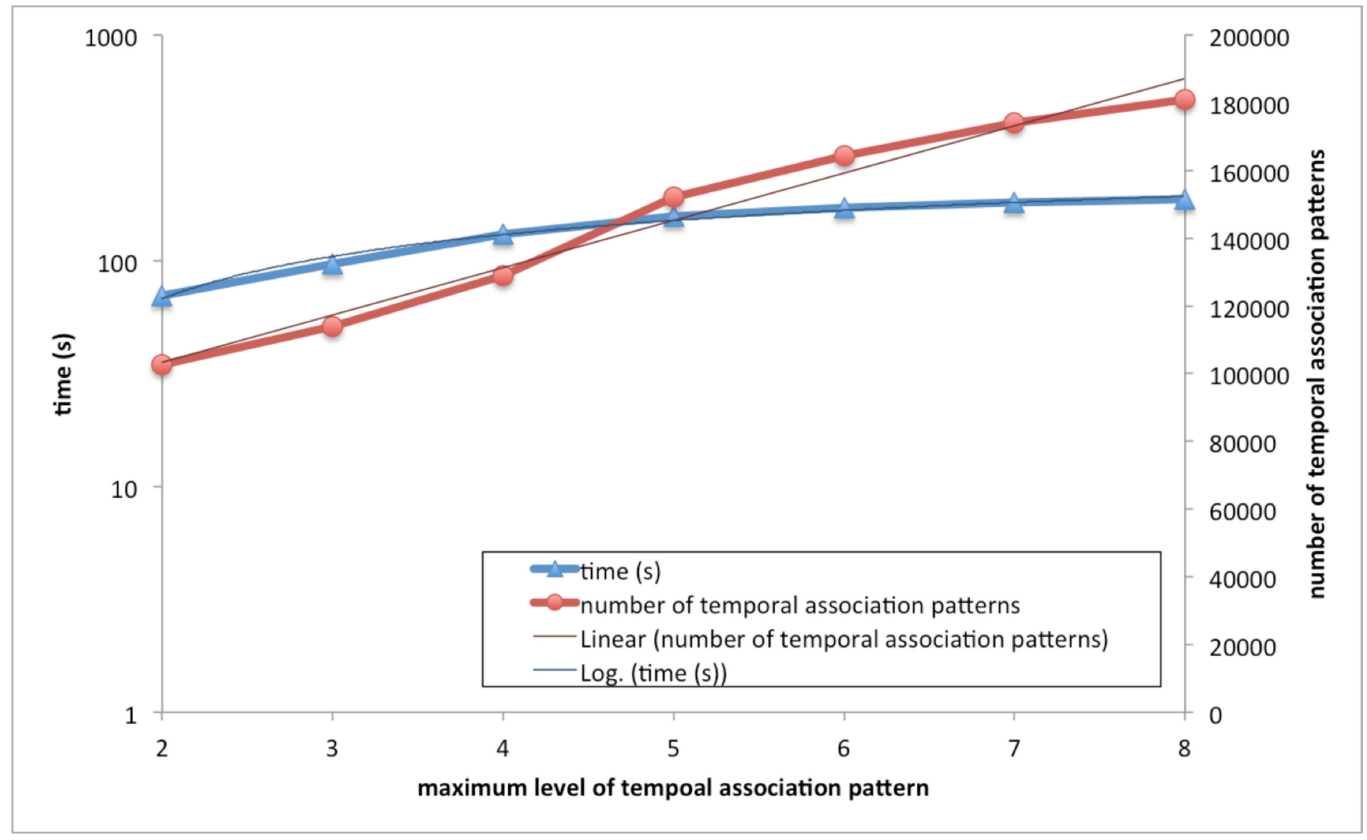

Fig. 3. Scalability results of the proposed algorithm.

Table 3. Top 9 Ranked Spatio-Temporal Patterns in Synthetic Data

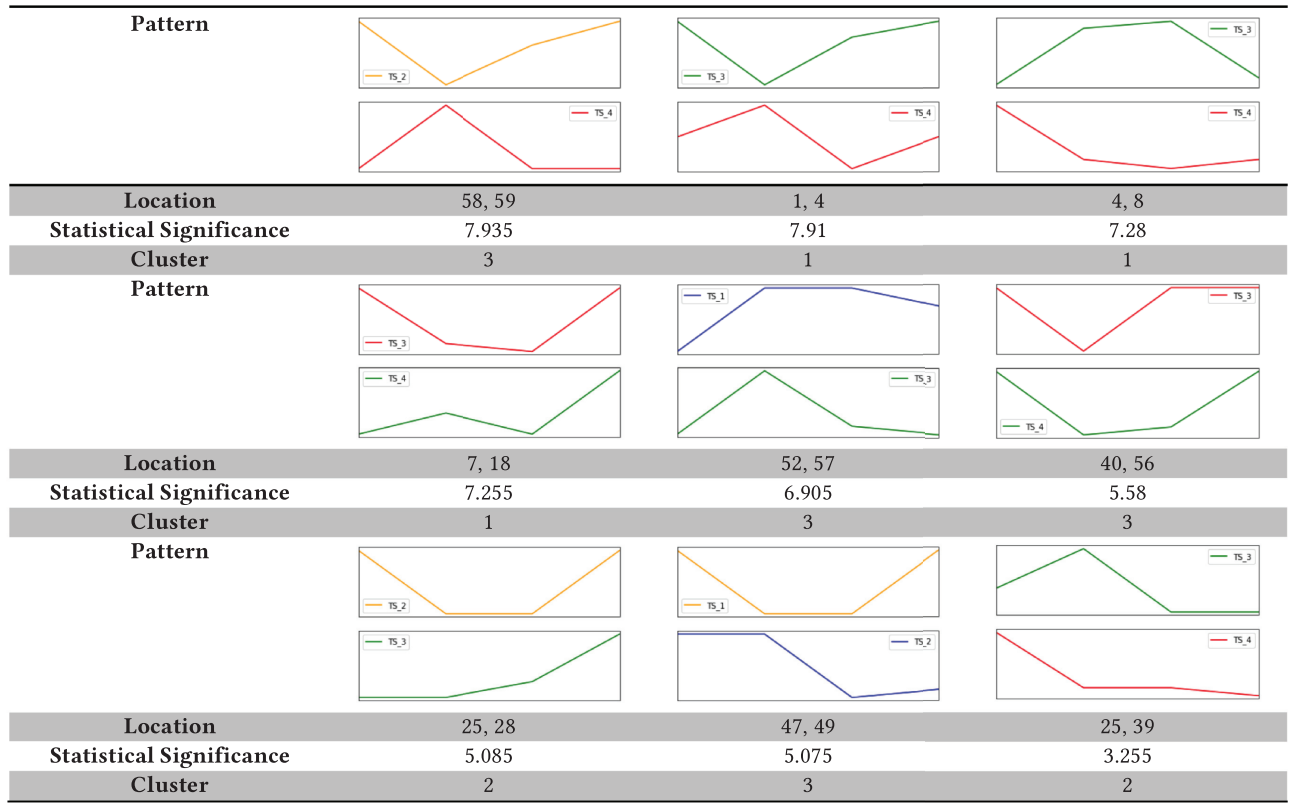


easier detection of the similarity of the regions. This analysis, therefore, justifies the importance of identifying spatio-temporal association patterns in MSTS database. For the STPM prepared by TARM's temporal patterns, it detected 11,360 spatio-temporal associations. Among these patterns, only up to the 224th ranked pattern is associated with the same cluster.

To evaluate the performance by clustering the generated STPM, given known cluster membership, we can calculate (i) F1-measure, defined as $F\left(C_{x}, C_{y}\right)=\frac{2 R\left(C_{x}, C_{y}\right) P\left(C_{x}, C_{y}\right)}{R\left(C_{x}, C_{y}\right)+P\left(C_{x}, C_{y}\right)}$, where $R\left(C_{x}, C_{y}\right)=$

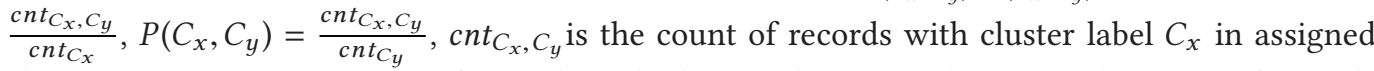
cluster $C_{y}, c n t_{C_{x}}$ is the count of records with cluster label $C_{x}$, and $c n t_{C_{y}}$ is the count of records in assigned cster $C_{y}$, and (ii) clustering accuracy, defined as $C A=\frac{\sum_{i=1}^{k} c_{n} t_{c^{i}}}{\text { Total number of records }}$, where cnt $_{c^{i}}$ is the count of records in $i$ th cluster and $k$ is the number of clusters.

To compare to the state of the art algorithms, we applied 3 model-based approaches to transform the MSTS into STPM, namely (a) PCA and ARMA, (b) PCA and equal frequency binning, and (c) lift ratio, and then perform clustering using $k$-means and HMM. PCA is used for feature extraction. These features are (a) modeled by ARMA to calculate the top eight LPC coefficients or (b) discretized into three intervals to form STPM. STPM is clustered by $k$-means $(k=3)$ using Euclidean distance or by HMM using log-likelihood value. The recent literature reported deep learning model based on CNN [32] could have achieved comparable performance to models fit on a version of the dataset with engineered features. Therefore, for comparison, we implemented (d) a one-dimensional convolutional neural network (1D CNN) model to classify the MSTS by inputting the cluster label given the known cluster membership in this synthetic dataset. Neural network classifier can be modified to fit a clustering model if a clustering algorithm such as $k$-means generates the cluster label initially and be it fed into the neural network's input layer. The network architecture is defined as having two 1D CNN layers, followed by a dropout layer for regularization, and then a pooling layer. The rectified linear unit (ReLU) function is chosen as the activation function that is a de-facto standard in recent deep learning models [33]. It is common to define $\mathrm{CNN}$ layers in groups of two to give the model a good chance of learning features from the input data. Practically, CNNs learn very quickly, so the dropout layer is intended to help slow down the learning process and hopefully result in a better final model. The pooling layer reduces the learned features to $1 / 4$ their size, consolidating them to only the most essential elements. After the CNN and pooling, the learned features are flattened to one long vector and pass through a fully connected layer before the output layer used to make a prediction. The fully connected layer ideally provides a buffer between the learned features and the output with the intent of interpreting the learned features before making a prediction. For this model, we use a standard configuration of 64 parallel feature maps and a kernel size of 3 . The feature maps are the number of times the input is processed or interpreted, whereas the kernel size is the number of input time steps considered as the input sequence is read or processed onto the feature maps. The efficient Adam version of stochastic gradient descent is used to optimize the network, and the categorical cross entropy loss function is used given that we are learning a multi-class classification problem. The model is fit for a fixed number of epochs, in this case, 10, and a batch size of 32 samples is used, where 32 windows of data is exposed to the model before the weights of the model are updated.

We split the data along the spatial dimension into $90 \%$ training set and $10 \%$ testing set. The result (Table 4) shows (e) the proposed approach outperforms the others. The first two approaches result in poor performance due to high information loss after dimensionality reduction. In (c), relationship between time series is considered so the hidden intra and inter patterns are revealed, significantly boosting the performance. In (d) given the ground-truth cluster label as an input for 
Table 4. Modelling and Clustering Comparison

\begin{tabular}{lcc}
\hline Approach & F1-measure & $C A$ \\
\hline (a) PCA + ARMA + k-means & 0.503 & $51.1 \%$ \\
(b) PCA + binning + HMM & 0.428 & $46.67 \%$ \\
(c) Lift ratio + k-means & 0.792 & $86.67 \%$ \\
d) CNN & 0.761 & $73.89 \%$ \\
(e) Proposed & 0.833 & $92.38 \%$ \\
\hline
\end{tabular}

CNN to learn a classifier, it is very robust to capture the temporal relationship that can yield better accuracy than (a) and (b). However, there is still room for improvement such as integrating different clustering methods into the network architecture while most existing deep learning-based clustering techniques have separate feature learning (via deep learning) and clustering (with traditional clustering methods). A recent attempt [34] is to simultaneously learn feature representation and does cluster assignment under the same deep learning framework on handwritten digit datasets and text datasets. However, it is not yet able to apply on multivariate spatial time-series data. The proposed approach not only considers important local associations between patterns temporally but also characterizes the space using only significant patterns globally, to produce high-quality matrix representation. Therefore, the noise from less significant patterns is filtered effectively and Pearson correlation coefficient, rather than Euclidean distance, is used for more effective clustering. Utilizing the information provided by the spatial penalty term, it also optimizes the proposed clustering algorithm to cluster the objects that share the common boundaries. Also, patterns hidden in each cluster are explicitly revealed and presented for easy interpretation even by a layperson.

\subsection{Experiments on North America Comprehensive Climate Dataset}

Comprehensive Climate Dataset (CCDS) is a collection of climate records with 125 observation locations using a $2.5 \times 2.5$ degree grid for latitudes in $(30.475,50.475)$ and longitudes in $(-119.75$, -79.75) from North America that contains monthly observations of 18 variables, including carbon dioxide $\left(\mathrm{CO}_{2}\right)$, methane $\left(\mathrm{CH}_{4}\right)$, carbon monoxide $(\mathrm{CO})$, hydrogen $\left(\mathrm{H}_{2}\right)$, wet days (WET), cloud cover (CLD), vapor (VAP), precipitation (PRE), frost days (FRS), diurnal temperature range (DTR), minimum temperate (TMN), average temperature (TMP), maximum temperature (TMX), global solar radiation (GLO), extraterrestrial radiation (ETR), extraterrestrial normal radiation (ETRN), direct solar radiation (DIR), and UV aerosol index (UV) from 1990 to 2002. The dataset can be obtained from Reference [28] and is a representative dataset used in spatial data mining field. In particular, the predictability of these variables has been revealed in the literature. These variables can be categorized as primary climate variables, and some human and natural variables such as solar irradiance and greenhouse gases as these are known to affect the climate. For further detail of these variables, see Reference [29].

In this experiment, we would extract the spatio-temporal patterns to characterize the CCDS by the STPM and then plot it on map (Figure 4) by the discovered patterns' statistical significance to demonstrate the ability to visualize them on different locations according to the strength of these patterns hidden in their climate measurements. Based on domain knowledge, we understand that past values of climate measurements in some specific locations to predict the future values of other time series are more predictive than the others. As a qualitative study, we will compare the strength of the discovered patterns by the proposed approach with the map of most predictive regions produced by Reference [28]. For the parameter setting, we set $\min _{o}$ to 5 based on the size of the dataset. We applied the proposed algorithms to form the STPM and extracted 31,256 spatio- 


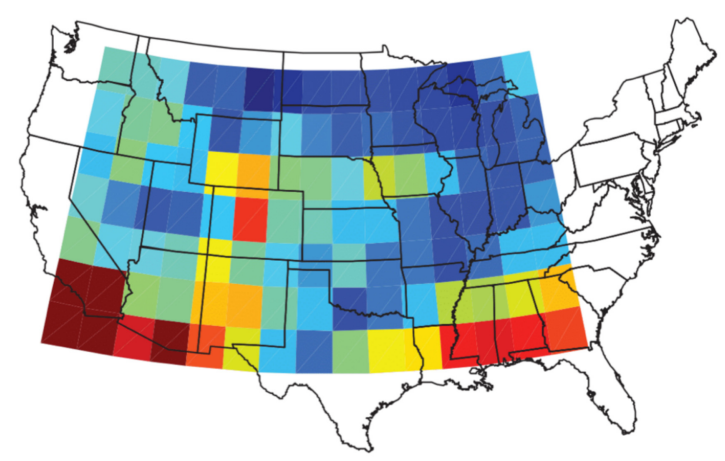

(a)

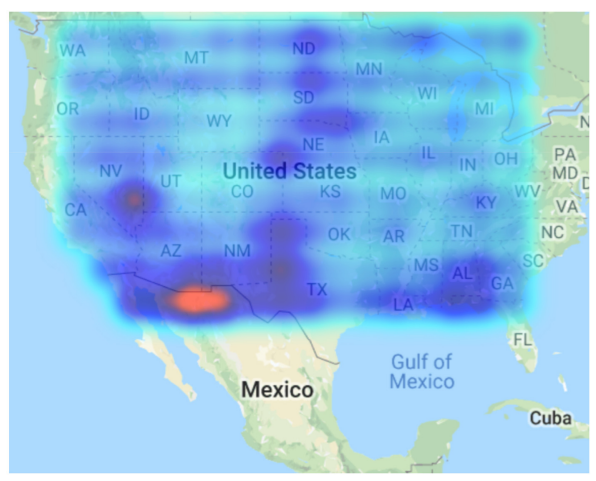

(b)

Fig. 4. (a) Map of the most predictive regions by reference [28] and (b) map of the most discriminative regions by the proposed algorithm from CCDS. On the left, red indicates highly predictive whereas blue indicates lowly predictive. On the right, light blue indicates weakly discriminative, whereas deep blue and red indicates strongly discriminative.

temporal patterns to represent the locations. To plot the map of most discriminative regions, we also define the aggregate pattern statistical significance of each region as $d^{\prime}(i)=\sum_{j}^{|S P|^{i}}\left|d_{S P j}\right|$, where $|S P|^{i}$ is the total number of patterns belonging to region $i$ and $d_{S P j}$ is the statistical significance of the $j^{t h}$ pattern in region $i$. Based on the ground truth, two regions are believed to be the most predictive regions that are the southwest and the southeast. The southwest region reflects the impact of the Pacific Ocean. Both color maps show the color level of southwest region, deep red in (a) and deep blue in (b), is stronger than other neighborhood regions. The southeast region frequently experiences relative sea level rise, hurricanes, and storm surge in the Gulf of Mexico. Again, on both color maps, the color level of southeast region suggests the past values are able to predict the future values of climate measurements and its discovered patterns are strongly discriminative than the neighborhood regions. The proposed approach can also reveal the interesting region in Colorado with both color maps showing high intensity of the values. It is because the Rocky Mountain valleys act as a funnel for winds from the west to provide locally divergent wind patterns. In short, this experiment can confirm the findings from the literature. It indicates and justifies the meaningfulness of the discovered spatio-temporal patterns that quantifies the temporal dependence between variables across different locations.

\subsection{A Case Study on Greater Bay Area Meteorological Dataset}

The Meteorological dataset (MET) is a collection of meteorological records taken from five different surface stations in the Greater Bay Area (GBA) in China that contains hourly observations over a 1-year-long period of eight attributes, including total cloudiness, lower cloudiness, dry bulb temperature, dew point temperature, relative humidity, site pressure, wind direction, and wind speed. These five surface stations denoted by the alphabets $S=\{A, B, C, D, E\}$ are located in the great urban region of GBA in China as shown in Figure 5. Stations A, B, C, D, and E are in Guangzhou metropolis, Foshan city, Shenzhen city, Dongguan city, and Zhongshan city, respectively. The description of data collected by each station is listed in Table 5. Based on the domain knowledge from Reference [24], all those meteorological variables have internal relationship according to the geographic location of the surface stations and might be governed by local terrain and land use.

We first selected the continuous variables for the MSTS analysis. In this case study, we set $\min _{o}$ to 300 based on the observation of the dataset. The proposed algorithms extracted 31,955 


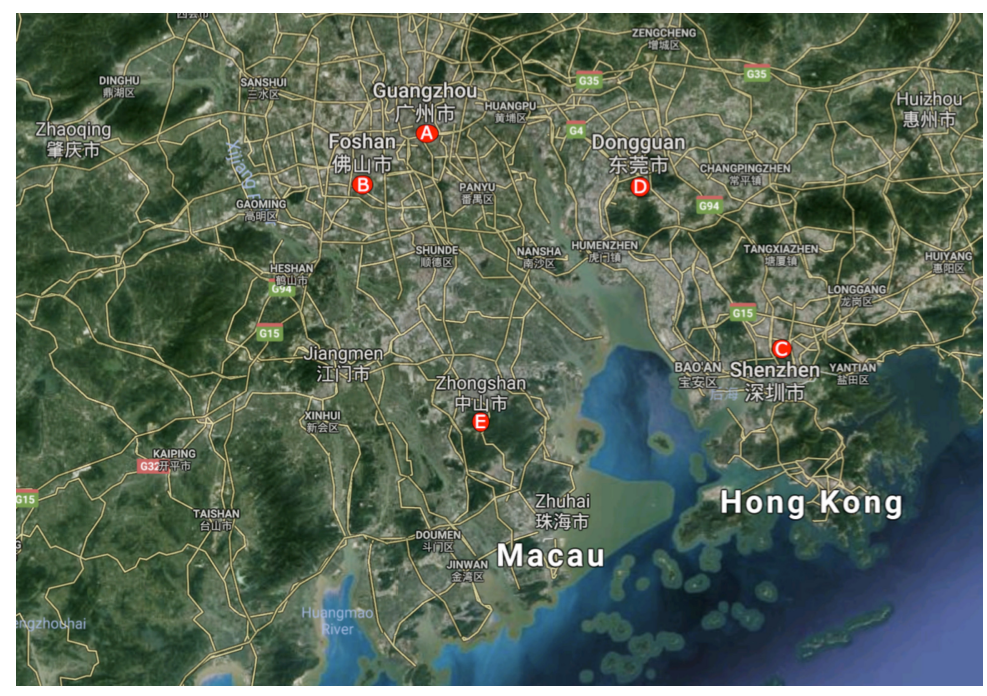

Fig. 5. Great Urban Region of Greater Bay Area (GBA) in China.

Table 5. Data Description of MET

\begin{tabular}{|ccc|}
\hline Attribute & Name & Type \\
\hline S1 & Total Cloudiness & Discrete \\
\hline S2 & Lower Cloudiness & Discrete \\
\hline S3 & Dry Bulb Temperature & Continuous \\
\hline S4 & Dew Point Temperature & Continuous \\
\hline S5 & Relative Humidity & Continuous \\
\hline S6 & Site Pressure & Continuous \\
\hline S7 & Wind Direction & Continuous \\
\hline S8 & Wind Speed & Continuous \\
\hline where $\mathbf{S}=\left\{\begin{array}{c}\text { A, B, }, \text { C, } \mathbf{D}\} \text { corresponds to a set of 5 } \\
\text { surface stations. }\end{array}\right.$ \\
\hline \multicolumn{3}{|c}{} \\
\hline
\end{tabular}

spatio-temporal patterns. Ranked by their statistical significance, we are able to reveal the patterns that are consistent to the attribute clustering from Reference [24] in which the top ranked patterns can distinguish the attribute cluster items. The attribute clustering by Reference [24] is able to identify the interdependence between attributes but is not able to make the temporal dependence hidden in the attributes across spaces explicit. The proposed algorithms can further uncover the movement of the values in the spatio-temporal dimension. Table 6 shows the top ranked spatio-temporal patterns for each attribute group formed according to Reference [24]. From the clusters labeled by domain experts, we understand that the grouping is based on the interdependence among similar characteristics of attributes within each cluster formed. The second, therd, and fourth patterns in which the location and attribute are exactly matched with the attribute clustering items reflect the regional (global) characteristics of the correlated meteorological parameters. For instance, an intra pattern in "A7" sensor for detecting wind direction in Guangzhou station significantly co-occurs in that in Foshan station. The first and fifth patterns reflect the local characteristics that are significantly influenced by the local geographical 
Table 6. Top Ranked Spatio-Temporal Patterns in MET

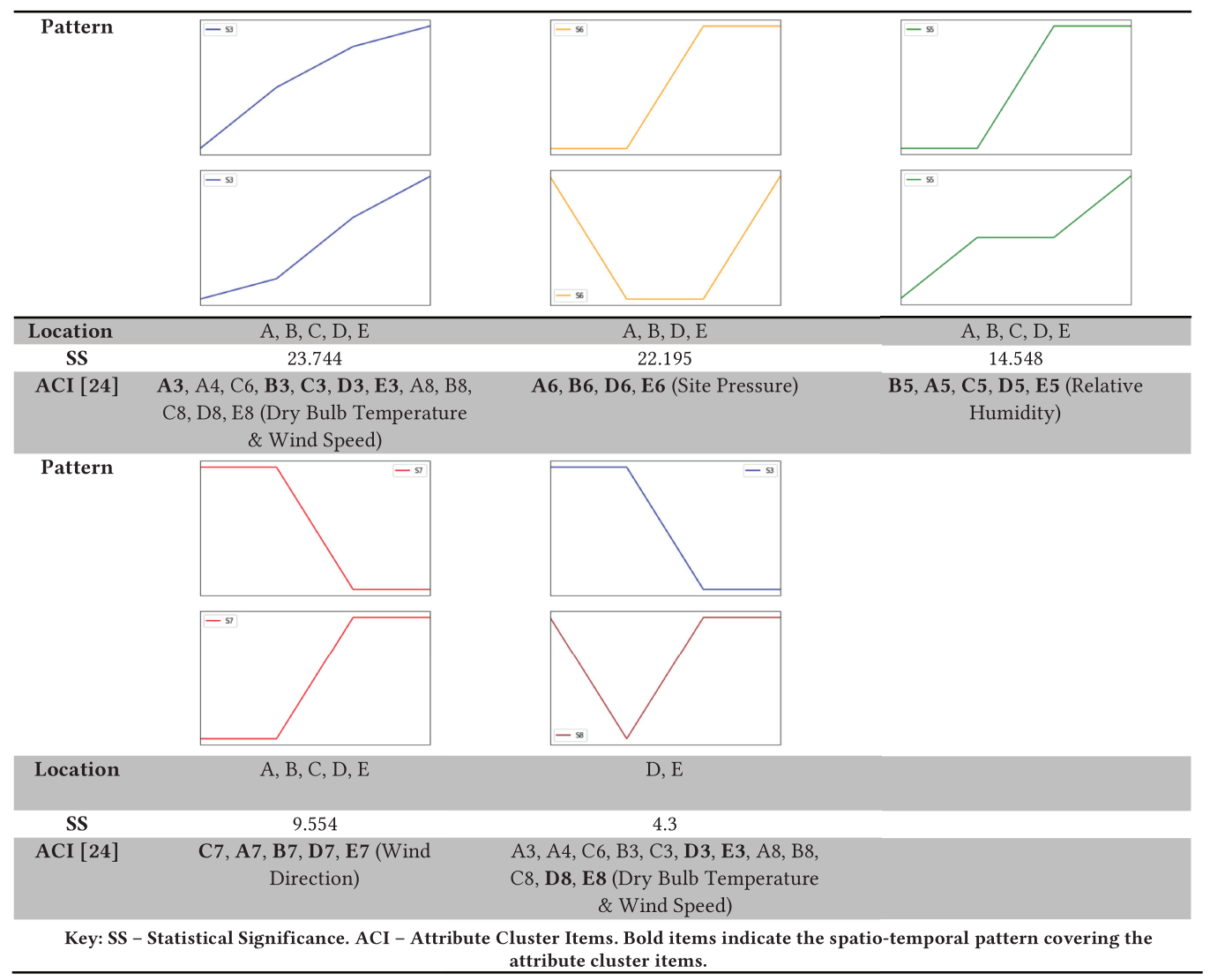

feature such as land use and land coverage. The fifth pattern is an inter pattern that consists of two different sensors for detecting dry bulb temperature and wind speed in Dongguan city and Zhongshan city. Comparing the values of statistical significance of the top five patterns, stations $\mathrm{A}, \mathrm{B}$, and $\mathrm{C}$ are in relatively stronger position than stations $\mathrm{D}$ and $\mathrm{E}$ for the weather condition analysis. This finding is corresponding to the claim in Reference [24] that the representative attributes in these attribute clusters are from only stations A, B, and C. Therefore, it justifies the meaningfulness of identifying the spatio-temporal patterns from the meteorological dataset.

\subsection{A Case Study on London Crime Dataset}

This section presents a case study on how our proposed approach has been used to assist police officers to analyze crime data. In the United Kingdom, police reported crimes are made publicly available via the police.uk website. The London crime dataset consists of monthly occurrences of 14 crime types, including anti-social behaviour, bicycle theft, burglary, criminal damage and arson, drugs, other crime, other theft, possession of weapons, public order, robbery, shoplifting, theft from the person, vehicle crime, violence and sexual offences, across 35 locations for latitudes in $(51.499,51.531)$ and longitudes in $(-0.227,0.554)$ in City of London spanning from September 2012 to August 2017. These 35 locations are formed according to the Lower Layer Super Output Area (LSOA), which is a geographic area built from groups of contiguous clusters of adjacent unit 


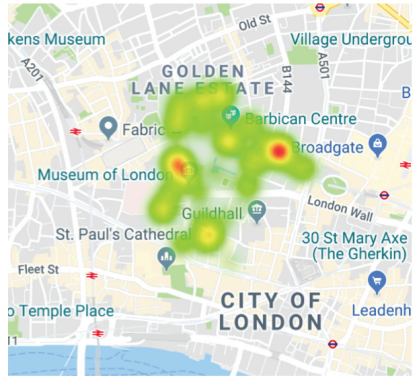

(a) Crime type: violence and sexual offences, vehicle crime

LSOA: City of London 001A, City of London 001B, City of London 001C, Tower Hamlets 015B

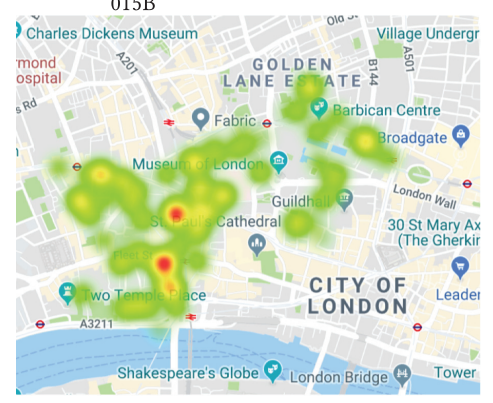

(d) Crime type: public order, vehicle crime

LSOA: City of London 001B, City of London 001C, City of London 001G

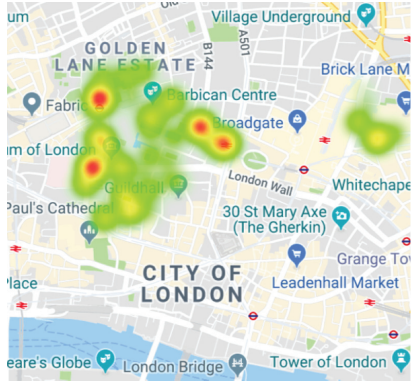

(b) Crime type: other theft, vehicle crime LSOA: City of London 001A, City of London 001B, City of London 001C, Tower Hamlets 015B

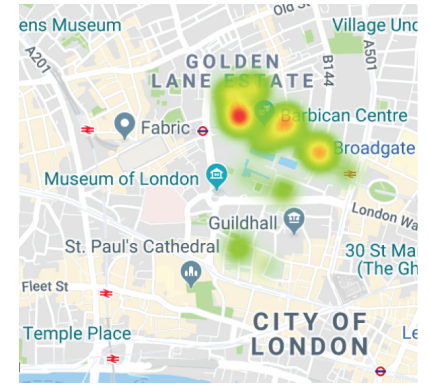

(c) Crime type: theft from the person, vehicle crime

LSOA: City of London 001B, City of London $001 \mathrm{C}$

Fig. 6. Color map of top five ranked patterns. Red color indicates the pattern occurrence is significantly frequent and the spatio-temporal association pattern is strongly discriminative. Green color indicates the pattern occurrence is also significantly frequent but the pattern is weekly discriminative. Yellow color indicates the pattern's strength is between those in red and green.

postcodes in the United Kingdom and is automatically generated to be as consistent in population size as possible. The LSOA codes and names are maintained by the Office for National Statistics in the United Kingdom. We will use the LSOA name to label the area in this case study.

In this case study, we set $\min _{o}=5,|\varepsilon|=3$ based on the size of the dataset. The proposed algorithms discovered 9,041 significant spatio-temporal association patterns in the study area. Ranked by the patterns' statistical significance, we plot the color map of the top five ranked patterns based on the values of the aggregate pattern statistical significance in Figure 6. The intensity of the color reflects the strength of the discovered patterns in the respective areas. From the color plot, it is obvious to identify some strong patterns as indicated by the red and yellow color. To further demonstrate the insight, we plot the transformed time series and highlight the association for the first ranked spatio-temporal pattern in Figure 7. This pattern consists of two crime types, violence and sexual offences as well as vehicle crime, across 4 LSOAs, namely City of London 001A, City of London 001B, City of London 001C and Tower Hamlets 015B. It reveals an interesting phenomenon that in these areas moderate number of occurrences of vehicle crime and low number of occurrences of violence and sexual offences are significantly correlated. By looking into the figures ending June 2017 from the Office for National Statistics [30], we found that vehicle-related thefts have increased in the City of London but sexual offences have declined. This kind of insight suggests police force work closely with partners in these areas with enforcement activity. The fact 


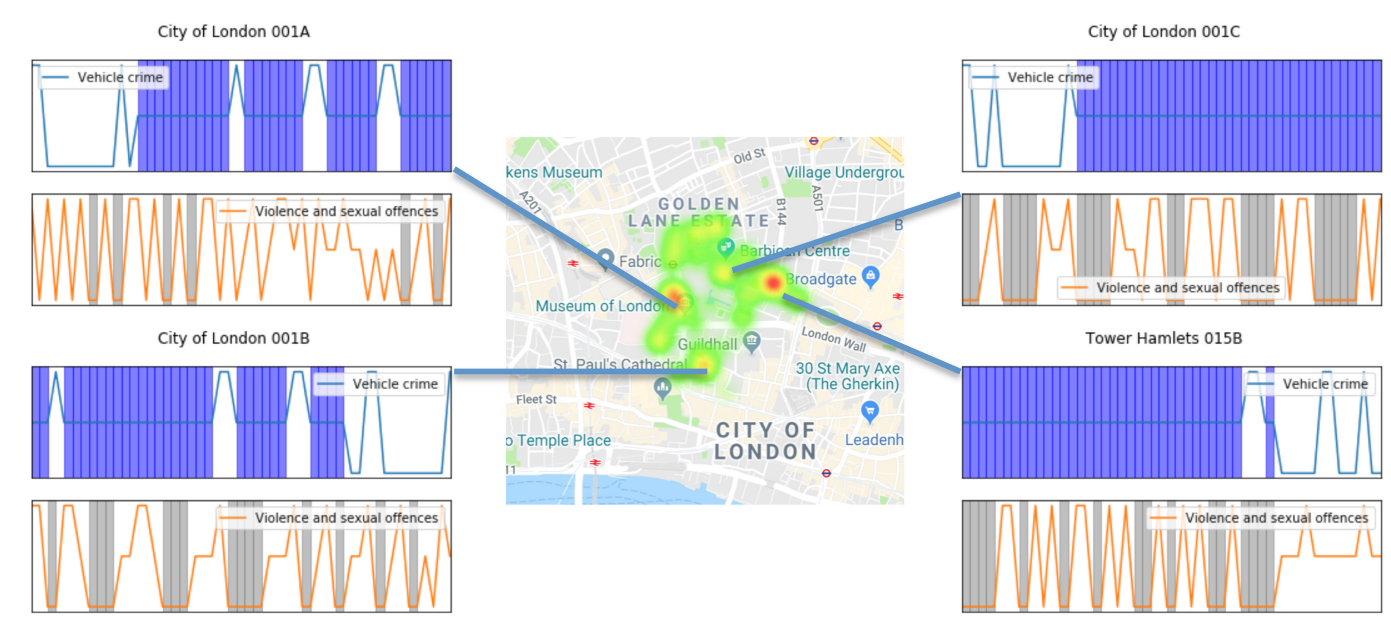

Fig. 7. The $1^{\text {st }}$ ranked spatio-temporal association in London crime data.

becomes evidence from this finding that justifies the importance and the interest for spatial and temporal pattern discovery from MSTS.

\section{CONCLUSION}

This article proposed a pattern mining approach to discover spatio-temporal associations for multivariate spatial time-series data. These associations capture patterns occurring sequentially with varying time delays in single and multiple time series across spaces. The matrix representation for the transformed MSTS stores important spatio-temporal pattern information to reveal the statistical significance, available for further clustering and classification. We have performed both experimental and case studies using the proposed approach. The experimental results have been consistent to the findings reported by the literature and show the proposed approach is able to uncover the relations among time series in multiple locations. The findings in the case studies may also provide previously unknown relations that could introduce interesting insight for subject matter experts. In addition, an aggregate statistical significance is introduced for ranking and visualizing the discovered patterns.

\section{REFERENCES}

[1] D. E. Zhuang, G. C. Li, and A. K. Wong. 2014. Discovery of temporal associations in multivariate time series. IEEE Trans. Knowl. Data Eng. 26, 12 (2014), 2969-2982.

[2] P. Y. Zhou and K. C. Chan. 2015. A feature extraction method for multivariate time series classification using temporal patterns. In Proceedings of the Pacific-Asia Conference on Knowledge Discovery and Data Mining. Springer, 409-421.

[3] A. M. MacEachren, M. Wachowicz, R. Edsall, D. Haug, and R. Masters. 1999. Constructing knowledge from multivariate spatiotemporal data: integrating geographical visualization with knowledge discovery in database methods. Int f. Geogr. Inf. Sci. 13, 4 (1999), 311-334.

[4] Z. Xing, J. Pei, and E. Keogh. 2010. A brief survey on sequence classification. ACM SIGKDD Explor. Newslett. 12, 1 (2010), 40-48.

[5] J. B. Kruskal and M. Liberman. 1983. The symmetric time warping problem: From continuous to discrete. In Time Warps, String Edits, and Macromolecules, 1983.

[6] T. Oates. 1999. Identifying distinctive subsequences in multivariate time series by clustering. In Proceedings of the 5th ACM SIGKDD International Conference on Knowledge Discovery and Data Mining. ACM, 322-326.

[7] C. Rosén and Z. Yuan. 2001. Supervisory control of wastewater treatment plants by combining principal component analysis and fuzzy c-means clustering. Water Sci. Technol. 43, 7 (2001), 147-156.

[8] K. Yang and C. Shahabi. 2004. A PCA-based similarity measure for multivariate time series. In Proceedings of the 2nd ACM International Workshop on Multimedia Databases. ACM, 65-74. 
[9] A. Singhal and D. E. Seborg. 2005. Clustering multivariate time-series data. f. Chemometr. 19, 8 (2005), 427-438.

[10] H. Yoon, K. Yang, and C. Shahabi. 2005. Feature subset selection and feature ranking for multivariate time series. IEEE Trans. Knowl. Data Eng. 17, 9 (2005), 1186-1198.

[11] L. M. Owsley, L. E. Atlas, and G. D. Bernard. 1997. Automatic clustering of vector time-series for manufacturing machine monitoring. In Proceedings of the IEEE International Conference on Acoustics, Speech, and Signal Processing (ICASSP'97) IEEE, 3393-3396.

[12] P. Y. Zhou and K. C. Chan. 2014. A model-based multivariate time series clustering algorithm. In Proceedings of the Pacific-Asia Conference on Knowledge Discovery and Data Mining. Springer, 805-817.

[13] R. Coppi, P. D’Urso, and P. Giordani. 2010. A fuzzy clustering model for multivariate spatial time series. f. Classif. 27, 1 (2010), 54-88.

[14] R. H. Shumway. 2005. Discrimination and clustering for multivariate time series. Encyclopedia of Biostatistics, 4.

[15] R. D. Pascual-Marqui. 2007. Instantaneous and lagged measurements of linear and nonlinear dependence between groups of multivariate time series: frequency decomposition. arXiv preprint arXiv:0711.1455

[16] P. Von Bünau, F. C. Meinecke, F. C. Király, and K.-R. Müller. 2009. Finding stationary subspaces in multivariate time series. Phys. Rev. Lett. 103, 21 (2009), 214101.

[17] A. F. Zuur, R. Fryer, I. Jolliffe, R. Dekker, and J. Beukema. 2003. Estimating common trends in multivariate time series using dynamic factor analysis. Environmetrics 14, 7 (2003), 665-685.

[18] M. Lavielle and G. Teyssiere. 2006. Detection of multiple change-points in multivariate time series. Lith. Math. 7. 46, 3 (2006), 287-306.

[19] R. S. Tsay, D. Peña, and A. E. Pankratz. 2000. Outliers in multivariate time series. Biometrika 87, 4 (2000), $789-804$.

[20] S. Frenzel and B. Pompe. 2007. Partial mutual information for coupling analysis of multivariate time series. Phys. Rev. Lett. 99, 20 (2007), 204101.

[21] A. Amiri-Simkooei. 2009. Noise in multivariate GPS position time-series. fournal of Geodesy 83, 2 (2009), 175-187.

[22] J. Lin, E. Keogh, L. Wei, and S. Lonardi. 2007. Experiencing SAX: A novel symbolic representation of time series. Data Min. Knowl. Discov. 15, 2, 107-144.

[23] S. J. Haberman. 1973. The analysis of residuals in cross-classified tables. Biometrics 29, 1, 205-220.

[24] A. K. Wong, B. Wu, G. P. Wu, and K. C. Chan. 2010. Pattern discovery for large mixed-mode database. In Proceedings of the 19th ACM International Conference on Information and Knowledge Management. ACM, 859-868.

[25] P. C. Ma, K. C. Chan, and D. K. Chiu. 2005. Clustering and re-clustering for pattern discovery in gene expression data. f. Bioinf. Comput. Biol. 3, 2 (2005), 281-301.

[26] Y. Wang and A. K. C. Wong. 2003. From association to classification: Inference using weight of evidence. IEEE Trans. Knowl. Data Eng. 15, 3 (2003), 764-767.

[27] G. Tatavarty, R. Bhatnagar, and B. Young. 2007. Discovery of temporal dependencies between frequent patterns in multivariate time series. In Proceedings of the IEEE Symposium on Computational Intelligence and Data Mining (CIDM'07). IEEE, 688-696.

[28] Y. Liu. 2015. Scalable multivariate time-series models for climate informatics. Computing in Science \& Engineering 17, 6 (2015), 19-26.

[29] A. C. Lozano, H. Li, A. Niculescu-Mizil, Y. Liu, C. Perlich, J. Hosking, and N. Abe. 2009. Spatial-temporal causal modeling for climate change attribution. In Proceedings of the 15th ACM SIGKDD International Conference on Knowledge Discovery and Data Mining. ACM, 587-596.

[30] J. Flatley. Crime in England and Wales: Year Ending June 2017. (October 2017). Retrieved July 11, 2018 from https:// www.ons.gov.uk/peoplepopulationandcommunity/crimeandjustice/bulletins/crimeinenglandandwales/june2017.

[31] M. L. Hetland and P. Sætrom. 2003. The role of discretization parameters in sequence rule evolution. In Proceedings of the International Conference on Knowledge-Based and Intelligent Information and Engineering Systems. Springer, Berlin, 518-525.

[32] J. Schmidhuber. 2015. Deep learning in neural networks: An overview. Neur. Netw. 61 (2015), 85-117.

[33] J. Gu, Z. Wang, J. Kuen, L. Ma, A. Shahroudy, B. Shuai, T. Liu, X. Wang, J. Cai, and T. Chen. 2018. Recent advances in convolutional neural networks. Pattern Recogn. 77 (2018), 354-377.

[34] K. Tian, S. Zhou, and J. Guan. 2017. Deepcluster: A general clustering framework based on deep learning. In Proceedings of the foint European Conference on Machine Learning and Knowledge Discovery in Databases. Springer, Cham, 809-825.

Received October 2018; revised September 2019; accepted November 2019 